\title{
Association between dietary patterns and adiposity from 4 to 7 years of age
}

\author{
Catarina Durão ${ }^{1}$, Milton Severo ${ }^{1,2}$, Andreia Oliveira ${ }^{1,2}$, Pedro Moreira ${ }^{1,3}$, \\ António Guerra ${ }^{4}$, Henrique Barros ${ }^{1,2}$ and Carla Lopes ${ }^{1,2, *}$ \\ 'EPIUnit - Institute of Public Health, University of Porto, Porto, Portugal: ${ }^{2}$ Department of Clinical Epidemiology, \\ Predictive Medicine and Public Health, Medical School, University of Porto, Alameda Prof. Hernâni Monteiro, \\ 4200-3 19 Porto, Portugal: ${ }^{3}$ Faculty of Nutrition and Food Sciences, University of Porto, Porto, Portugal: \\ ${ }^{4}$ Department of Paediatrics, Medical School, University of Porto, Porto, Portugal
}

Submitted 16 December 2016: Final revision received 3 April 2017: Accepted 7 April 2017: First published online 23 May 2017

\begin{abstract}
Objective: The present study aimed to evaluate the association of 4-year-old children's dietary patterns with adiposity at 7 years, according to child's sex, using a conceptual model.

Design: Prospective cohort study. Diet was assessed using an FFQ. Age- and sex-specific BMI standard deviation scores (Z-scores) were defined according to the WHO. Fat mass percentage (FM\%), fat mass index (FMI) and waist-to-height ratio (WHtR) were also considered, converted into $Z$-scores using sex-specific means and standard deviations of the current sample. Dietary patterns were identified by latent class analysis and their association with adiposity was estimated by linear regression models.

Setting: Population-based birth cohort Generation XXI (Porto, Portugal, 2005-2006). Subjects: Children ( $n$ 3473) evaluated at both 4 and 7 years of age.

Results: Three dietary patterns were identified: high in energy-dense foods (EDF); low in foods typically consumed at main meals and intermediate in snacks (Snacking); and higher in vegetables and fish and lower in EDF (Healthier, reference). The EDF dietary pattern at 4 years of age was positively associated with later BMI only in girls $(\beta=0 \cdot 075,95 \%$ CI 0.009, 0.140, $P$-interaction $=0 \cdot 046)$. The EDF dietary pattern was also associated with other adiposity indicators only in girls (FMI: $\beta=0 \cdot 071,95 \%$ CI 0.000, 0.142; WHtR: $\beta=0 \cdot 094,95 \%$ CI 0.023, $0 \cdot 164$ ). Snacking was not significantly associated with any marker of adiposity in either girls or boys.

Conclusions: Although dietary patterns and adiposity persisted across the two ages in both sexes, EDF at 4 years of age increased adiposity at 7 years of age only in girls.
\end{abstract}

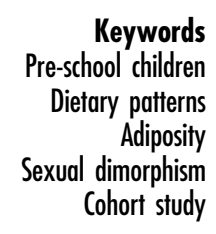

Although the prevalence of childhood obesity might be reaching a plateau in some countries ${ }^{(1,2)}$, it has generally increased in the last four decades and remains high ${ }^{(3)}$. In Europe, the prevalence of overweight in children below 10 years of age is higher in southern countries and differences by sex have been reported ${ }^{(4)}$. In Portugal ${ }^{(5)}$, considering either Cole and Lobstein's ${ }^{(6)}$ or the WHO's ${ }^{(7)}$ criteria, prevalence of overweight among 7-year-olds is higher in girls than in boys.

Research on dietary determinants of childhood obesity has focused mainly on specific nutrients and foods, but this approach does not account for the complexity of the human diet. Analysis of dietary patterns considers both interactions between foods and clustered dietary behaviours $^{(8-10)}$ and may better capture the overall effect of dietary intake, including total energy intake, on adiposity, helping to better understand the relationship between diet and disease. Besides energy intake, the effect of nutrients on adiposity may also be better captured by overall measures of dietary intake, and both energy and nutrient intakes might have sex-specific effects on adiposity as suggested in a recent study showing that the effect of energy intake was independent of macronutrient distribution in girls, but not in boys ${ }^{(11)}$.

Prospective studies examining the association of empirically derived dietary patterns practised at pre-school age with adiposity are scarce ${ }^{(12,13)}$ and have not found an association from 3 to 7 years or from 5 to 9 years of age. Methodological aspects in the identification of dietary patterns, such as subjective cut-off points on underlying 
dimensions, may not allow identification of all dietary patterns in a data set. Hence, using a distinct methodology that avoids subjective decisions, such as latent class analysis ${ }^{(14)}$, might be advantageous to explore unobserved variables (i.e. latent classes) that distinguish homogeneous clusters of individuals (e.g. dietary patterns) in a data set.

In children, sexual dimorphism is observed from an early age in both total and regional adiposity ${ }^{(15-17)}$ and hormone biology (i.e. insulin and leptin). Yet, prospective studies have not reported formal examination of an interaction of child's sex on the association between dietary patterns of pre-school children and adiposity. In addition, considering sexual dimorphism in BMI trajectories - with girls showing older age and lower BMI at the infancy peak and earlier adiposity rebound ${ }^{(18,19)}$ - and keeping in mind that sex differences in the susceptibility to environmental factors such as peer influence and restrictive parenting practices have been reported ${ }^{(20)}$, distinct prospective associations of dietary patterns and adiposity in girls and boys could be possible.

Considering the paucity of evidence, that eating habits start to be established in early years tracking into later life ${ }^{(21,22)}$ and that the evidence base for intervention at preschool age is scarce ${ }^{(23)}$, prospective studies on pre-school children's dietary patterns and adiposity, taking into account child's sex, are particularly warranted. Hence, the aim of the present study was to evaluate if dietary patterns of 4-year-old children were associated with adiposity at 7 years of age, using a conceptual model to examine hypothesized sex-specific prospective associations.

\section{Methods}

\section{Subjects}

The present study was based on the population-based birth cohort Generation XXI (Porto, Portugal, 2005-2006), described elsewhere ${ }^{(24)}$. At child's age of 4 years, the entire cohort was invited to a re-evaluation conducted as previously outlined $^{(25,26)}$ and, at 7 years, a new follow-up of the whole cohort took place ( $81 \%$ participation). The present study considered a sub-sample of singleton children with information on body composition measured at both ages ( $n 3854$ ). After exclusion of children with diseases that might influence dietary intake (e.g. cerebral palsy or food allergy, $n 17$ ), with missing information on dietary intake ( $n 46$ and $n 81$ at 4 and 7 years, respectively) and those with incomplete information on potential confounders (exercise, $n 12$; maternal weigh/height, $n 225), 3473$ children were included in final analyses.

\section{Dietary intake}

Dietary intake was evaluated by an FFQ adapted from a previous questionnaire validated for the general adult population $^{(27)}$, shortened for application in the current birth cohort. The FFQ was applied by trained staff to the child's main caregiver at both waves and queried about how many times, on average, children consumed thirty-five food items during the previous 6 months. Details on response options and conversion into daily frequencies are described elsewhere ${ }^{(26)}$. In a sub-sample of 2373 children with complete $3 \mathrm{~d}$ food diaries, the validity and reliability of the FFQ were assessed by Pearson's correlation coefficients and intraclass correlation coefficients for key food groups as measured by both methods. For foods most frequently consumed, weak-tomoderate correlations and fair-to-moderate agreement were observed. Significant positive moderate Pearson's correlations were found for vegetable soup $(r=0.54$, $P<0.001)$, fruit $(r=0.42, \quad P<0.001)$, milk $\quad(r=0.46$, $P<0.001)$ and yoghurts $(r=0.48, P<0.001)$. Intraclass correlation coefficients varied from 0.54 (95\% CI 0.51 , $0.56)$ for vegetable soup to 0.17 (95\% CI $0.11,0.32)$ for juices. In addition, $3 \mathrm{~d}$ food diaries showed that there were no severe outliers $(3 \times$ interquartile range) in daily energy intake in either girls or boys.

As detailed earlier ${ }^{(28)}$, sixteen food items/groups (i.e. milk, yoghurt, meat/eggs, sweets, soft drinks, fruit, vegetable soup, vegetables, cheese, fish, processed meat, rice/pasta/potatoes, bread, crisps, pizza/burger, salty pastry) were used to identify dietary patterns at 4 years. Due to low variability in frequency of consumption of some foods, items/groups were categorized into 1st quintile, 2nd to 4 th quintiles combined and 5 th quintile or into two categories $(1 / \mathrm{d}, 2 / \mathrm{d})$ for foods eaten only once or twice daily (e.g. vegetable soup). Because, at 4 years of age, the cut-off points for consumption categories were very similar in girls and boys, the cut-off points of the whole cohort were used to identify dietary patterns. The same sixteen items/groups and cut-off points observed at 4 years were defined at 7 years of age. This approach was defined a priori to examine if children changed dietary pattern from 4 to 7 years, rather than to identify new patterns at 7 years. To obtain the dietary patterns at 7 years of age, we calculated the posterior probability of children belonging to each latent class from the parameters estimated by the latent class model at 4 years, considering the food group variables from 7 years.

\section{Anthropometric data}

Child's weight and height were measured at both ages as previously described ${ }^{(26)}$. Age- and sex-specific BMI standard deviation scores (BMI $Z$-scores) were computed according to the $\mathrm{WHO}^{(7,29)}$. Fat mass was assessed by tetrapolar bioelectric impedance (BIA 101 Anniversary ${ }^{\circledR}$; Akern, Florence, Italy) according to standard procedures ${ }^{(30)}$ and equipment outputs were converted into fat-free mass ${ }^{(31)}$ from which fat mass percentage (FM\%) and fat mass index (FMI; $\mathrm{kg} / \mathrm{m}^{2}$ ) were derived. Waist circumference was measured at the umbilicus level ${ }^{(32)}$ and waist-to-height ratio (WHtR) was calculated. To improve comparability, FMI, FM\% and WHtR were converted into $Z$-score units 
using the sex-specific means and standard deviations observed in the sample.

\section{Potential confounders}

Potential confounders were selected based on both previous literature and statistical methods, as described in the next section.

Maternal BMI and completed schooling years at child's age of 4 years were included as continuous variables in all analyses. Maternal work status was categorized into not working $v$. working and maternal smoking status was categorized into smoking $v$. no.

Child's exact age was considered in years. Any breastfeeding was categorized into $<6 v . \geq 6$ months. Regular practice of structured physical exercise was considered as a dichotomous variable (no $v$. yes) and screen time was considered as $>2 v . \leq 2 \mathrm{~h} / \mathrm{d}$.

\section{Statistical analysis}

Considering the categorical nature of the response items in the FFQ and their asymmetric distribution, children's dietary patterns were identified by latent class analysis ${ }^{(14)}$. This methodology is a person-centred approach, used to distinguish homogeneous clusters of individuals from a sample $^{(14,33)}$. In order to avoid selection of spurious latent classes (type I error) that is more common when using powerful tests such as the Lo-Mendell-Rubin likelihood ratio test, in the present study the number of dietary patterns was defined according to the Bayesian information criterion, which is a criterion that performs well in large sample sizes ${ }^{(34)}$. Individuals were assigned to each pattern according to the highest probability of class membership and probabilities of choosing each item response (e.g. consumption categories), conditionally on class membership, can be interpreted based on item profiles in each cluster ${ }^{(33,35)}$. As energy intake can be an intermediate step in the pathway between diet and adiposity, dietary patterns were not adjusted for energy.

The association of dietary patterns with adiposity was assessed by linear regression coefficients $(\beta)$ and respective $95 \%$ CI. Four separate models (BMI, FM\%, FMI and WHtR) were fitted. Confounding factors were assessed in each model and those not associated with the outcome or that did not change the association of interest were not considered in the final models (maternal age, work status and smoking habits). For theoretical reasons, children's practice of physical exercise, daily screen time and breast-feeding were included in all models. Furthermore, as energy intake was considered a possible intermediate step in the pathway between dietary patterns and adiposity, models were not adjusted for it. The interaction of child's sex on the association with adiposity was examined by a product term added into fully adjusted models.

In order to assess relationships of diet and adiposity across time, an analysis based on a theoretical concept (Fig. 1) was also conducted, including three components: (i) a crossed analysis of exposure at time 1 on outcome at

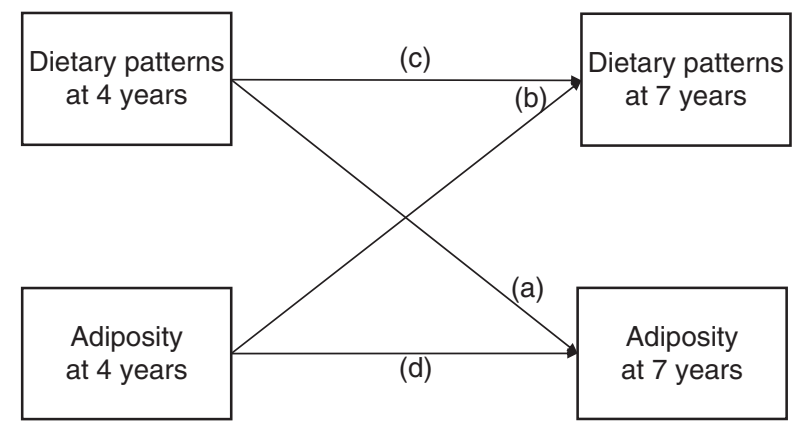

Fig. 1 Prospective relationships were analysed considering associations of: (a) dietary patterns at 4 years with adiposity at 7 years (results in Table 3); (b) adiposity at 4 years with later dietary patterns (results in Table 4); (c) dietary patterns across ages (results in Table 5); and (d) adiposity across time (results in Table 6)

time 2 (i.e. the main association of interest, denoted (a) in Fig. 1) and of outcome at time 1 on exposure at time 2 (denoted (b) in Fig. 1); (ii) an analysis of the exposure across time (denoted (c) in Fig. 1); and (iii) an analysis of the outcome across the two ages (denoted (d) in Fig. 1).

Associations of adiposity measures across time were assessed by linear regression models. Associations of adiposity at 4 years with later dietary pattern, as well as of patterns across the two ages, were assessed by OR and respective $95 \% \mathrm{CI}$ estimated by multinomial logistic regression models. In linear regression models homoscedasticity of the error and normality of error distribution were assessed, while in multinomial logistic regression models goodness-of-fit was assessed by Pearson's $\chi^{2}$ tests.

Statistical analyses were conducted using the statistical software package IBM SPSS Statistics version 22 and Mplus version 5.2 (Muthén \& Muthén, Los Angeles, CA, USA), and a significance level of $5 \%$ was considered.

\section{Results}

Three dietary patterns were identified at 4 years of age (Table 1): (i) a pattern characterized by higher proportions of children within the 5th quintile for sweets, soft drinks, salty pastry and processed meat (named 'Energy-Dense Foods' (EDF)); (ii) a pattern lower in foods typically eaten at main meals (e.g. vegetables on a plate, fish, meat and eggs, rice/ pasta/potatoes) and intermediate in snacks (named 'Snacking'); and (iii) a dietary pattern higher in healthy foods and lower in unhealthier ones (named 'Healthier' and used as reference category). At 4 years, $43.7 \%$ of girls and $44.5 \%$ of boys followed the EDF dietary pattern, while at 7 years (Table 2) proportions increased to 48.8 and $52.7 \%$, respectively. In contrast, proportions of children following the Snacking pattern decreased from 4 to 7 years of age in both sexes. Likewise, class membership in the Healthier dietary pattern decreased. 
Table 1 Dietary patterns at 4 years of age: proportions of children in lower and higher consumption categories by sex $(n 3473)^{\star}$, Generation XXI prospective cohort study (Porto, Portugal, 2005-2006)

\begin{tabular}{|c|c|c|c|c|c|c|c|c|}
\hline \multirow[b]{2}{*}{ Item/category $†$} & \multicolumn{4}{|c|}{ Girls } & \multicolumn{4}{|c|}{ Boys } \\
\hline & $\begin{array}{l}\text { Total sample } \\
(n 1722)\end{array}$ & $\begin{array}{c}\text { LC1 } \\
\text { (EDF) } \\
(n 753)\end{array}$ & $\begin{array}{c}\text { LC2 } \\
\text { (Snacking) } \\
(n 215)\end{array}$ & $\begin{array}{c}\text { LC3 } \\
\text { (Healthier) } \\
(n 754)\end{array}$ & $\begin{array}{c}\text { Total sample } \\
(n 1751)\end{array}$ & $\begin{array}{c}\text { LC1 } \\
\text { (EDF) } \\
(n 780)\end{array}$ & $\begin{array}{c}\text { LC2 } \\
\text { (Snacking) } \\
(n 250)\end{array}$ & $\begin{array}{c}\text { LC3 } \\
\text { (Healthier) } \\
(n 721)\end{array}$ \\
\hline \multicolumn{9}{|l|}{ Fruit } \\
\hline 1st quintile & 39.9 & $54 \cdot 7^{\mathrm{a}}$ & $60 \cdot 0^{a}$ & $19 \cdot 4^{b}$ & 39.4 & $50 \cdot 1^{a}$ & $68.0^{\mathrm{b}}$ & $17 \cdot 9^{\mathrm{c}}$ \\
\hline 5th quintile & 2.7 & $2 \cdot 4^{a}$ & $0.9^{a}$ & $3 \cdot 4^{\mathrm{a}}$ & $2 \cdot 3$ & $2 \cdot 6^{a}$ & $0.8^{a}$ & $2 \cdot 6^{a}$ \\
\hline \multicolumn{9}{|l|}{ Vegetable soup } \\
\hline$\leq 1 / d$ & $25 \cdot 4$ & $36 \cdot 1^{a}$ & $30 \cdot 2^{a}$ & $13 \cdot 3^{b}$ & 24.5 & $35 \cdot 8^{a}$ & $25 \cdot 6^{b}$ & $11.9^{c}$ \\
\hline$\geq 2 / d$ & 74.6 & $63.9^{\mathrm{a}}$ & $69 \cdot 8^{\mathrm{a}}$ & $86 \cdot 7^{\mathrm{a}}$ & 75.5 & $64 \cdot 2^{a}$ & $74.4^{\mathrm{b}}$ & $88.1^{\mathrm{C}}$ \\
\hline \multicolumn{9}{|c|}{ Vequtables on a plate } \\
\hline 1st quintile & 28.9 & $28.8^{\mathrm{a}}$ & $80.0^{\mathrm{b}}$ & $14.5^{\mathrm{c}}$ & $32 \cdot 8$ & $30 \cdot 6^{a}$ & $81.6^{\mathrm{b}}$ & $18 \cdot 2^{\mathrm{C}}$ \\
\hline 5th quintile & $21 \cdot 3$ & $17 \cdot 7^{\mathrm{a}}$ & $4 \cdot 2^{b}$ & $29 \cdot 8^{c}$ & $20 \cdot 2$ & $15 \cdot 3^{a}$ & $3 \cdot 6^{b}$ & $31 \cdot 2^{\mathrm{c}}$ \\
\hline \multicolumn{9}{|l|}{ Milk } \\
\hline 1st quintile & $21 \cdot 3$ & $21 \cdot 2^{\mathrm{a}}$ & $23 \cdot 3^{\mathrm{a}}$ & $20 \cdot 7^{a}$ & $18 \cdot 7$ & $19 \cdot 6^{a}$ & $17 \cdot 2^{\mathrm{a}}$ & $18 \cdot 2^{\mathrm{a}}$ \\
\hline 5th quintile & 8.0 & $6 \cdot 9^{a}$ & $14 \cdot 4^{b}$ & $7 \cdot 2^{a}$ & 9.9 & $10 \cdot 4^{a, b}$ & $13 \cdot 6^{b}$ & $8 \cdot 2^{a}$ \\
\hline \multicolumn{9}{|l|}{ Yoghurt } \\
\hline 1st quintile & $57 \cdot 7$ & $50 \cdot 6^{a}$ & $46 \cdot 5^{a}$ & $68 \cdot 0^{b}$ & 50.5 & $43 \cdot 2^{a}$ & $40 \cdot 0^{a}$ & $62 \cdot 1^{b}$ \\
\hline 5th quintile & 4.5 & $5 \cdot 2^{a}$ & $4 \cdot 7^{\mathrm{a}}$ & $3 \cdot 8^{\mathrm{a}}$ & 5.6 & $7 \cdot 1^{a}$ & $5 \cdot 2^{a, b}$ & $4 \cdot 2^{b}$ \\
\hline \multicolumn{9}{|l|}{ Cheese } \\
\hline 1st quintile & 24.5 & $16 \cdot 3^{a}$ & $64 \cdot 7^{b}$ & $21 \cdot 2^{c}$ & $23 \cdot 4$ & $16 \cdot 8^{a}$ & $48 \cdot 8^{b}$ & $21 \cdot 6^{a}$ \\
\hline 5 th quintile & 8.1 & $10 \cdot 5^{\mathrm{a}}$ & $7 \cdot 0^{a, b}$ & $6 \cdot 1^{\mathrm{b}}$ & 7.1 & $10 \cdot 5^{a}$ & $7 \cdot 6^{\mathrm{a}}$ & $3 \cdot 2^{b}$ \\
\hline \multicolumn{9}{|l|}{ Fish } \\
\hline 1st quintile & 67.5 & $78 \cdot 5^{a}$ & $82 \cdot 3^{a}$ & $52 \cdot 3^{b}$ & $67 \cdot 0$ & $77 \cdot 7^{\mathrm{a}}$ & $90 \cdot 0^{b}$ & $47 \cdot 6^{c}$ \\
\hline 5th quintile & $7 \cdot 7$ & $3 \cdot 3^{\mathrm{a}}$ & $5 \cdot 1^{\mathrm{a}}$ & $12 \cdot 7^{b}$ & $9 \cdot 1$ & $4 \cdot 2^{\mathrm{a}}$ & $4 \cdot 8^{\mathrm{a}}$ & $16 \cdot 0^{\mathrm{b}}$ \\
\hline \multicolumn{9}{|l|}{ Meat and eggs } \\
\hline 1st quintile & 39.8 & $40 \cdot 6^{a}$ & $51 \cdot 6^{b}$ & $35 \cdot 7^{a}$ & $37 \cdot 2$ & $34 \cdot 7^{\mathrm{a}}$ & $46 \cdot 8^{b}$ & $36 \cdot 6^{a}$ \\
\hline 5th quintile & $17 \cdot 7$ & $20 \cdot 1^{a}$ & $8 \cdot 4^{\mathrm{b}}$ & $18 \cdot 0^{\mathrm{a}}$ & $18 \cdot 0$ & $19 \cdot 7^{a}$ & $12 \cdot 8^{b}$ & $17 \cdot 9^{a, b}$ \\
\hline \multicolumn{9}{|c|}{ Processed meat } \\
\hline 1st quintile & $17 \cdot 4$ & $4.4^{\mathrm{a}}$ & $39.5^{\mathrm{b}}$ & $24.1^{\mathrm{c}}$ & 21.6 & $6 \cdot 4^{\mathrm{a}}$ & $58 \cdot 4^{\mathrm{b}}$ & $25 \cdot 4^{\mathrm{c}}$ \\
\hline 5th quintile & $20 \cdot 0$ & $29 \cdot 2^{a}$ & $15 \cdot 8^{b}$ & $11.9^{\mathrm{b}}$ & $18 \cdot 8$ & $27 \cdot 6^{\mathrm{a}}$ & $10 \cdot 8^{b}$ & $12 \cdot 1^{\mathrm{b}}$ \\
\hline \multicolumn{9}{|c|}{ Rice/potatoes/pasta } \\
\hline$\leq 1 / d$ & 7.4 & $6 \cdot 4^{\mathrm{a}}$ & $16 \cdot 7^{\mathrm{b}}$ & $5 \cdot 8^{a}$ & 8.3 & $6 \cdot 7^{\mathrm{a}}$ & $19 \cdot 6^{\mathrm{b}}$ & $6 \cdot 2^{\mathrm{a}}$ \\
\hline$\geq 2 / d$ & 92.6 & $93 \cdot 6^{\mathrm{a}}$ & $83 \cdot 3^{b}$ & $94 \cdot 2^{\mathrm{a}}$ & 91.7 & $93 \cdot 3^{a}$ & $80 \cdot 4^{b}$ & $93 \cdot 8^{a}$ \\
\hline \multicolumn{9}{|l|}{ Bread } \\
\hline 1st quintile & $51 \cdot 4$ & $47 \cdot 5^{\mathrm{a}}$ & $51 \cdot 6^{a, b}$ & $55 \cdot 2^{b}$ & $46 \cdot 4$ & $43 \cdot 6^{a}$ & $49 \cdot 2^{a}$ & $48 \cdot 4^{a}$ \\
\hline 5 th quintile & 1.4 & $1 \cdot 7^{\mathrm{a}}$ & $1.9^{a}$ & $0.9^{a}$ & 1.8 & $2 \cdot 2^{a}$ & $1.6^{a}$ & $1.4^{\mathrm{a}}$ \\
\hline \multicolumn{9}{|l|}{ Crisps } \\
\hline 1st quintile & $23 \cdot 4$ & $7 \cdot 3^{a}$ & $24 \cdot 7^{b}$ & $39 \cdot 1^{c}$ & $23 \cdot 1$ & $9 \cdot 0^{\mathrm{a}}$ & $22 \cdot 4^{b}$ & $38 \cdot 6^{c}$ \\
\hline 5th quintile & 8.9 & $16 \cdot 5^{a}$ & $12 \cdot 6^{b}$ & $0.4^{c}$ & 8.7 & $16 \cdot 5^{a}$ & $9 \cdot 6^{\mathrm{b}}$ & $0.0^{\mathrm{C}}$ \\
\hline \multicolumn{9}{|l|}{ Pizza/burger } \\
\hline 1st quintile & $24 \cdot 2$ & $8 \cdot 0^{\mathrm{a}}$ & $62 \cdot 8^{b}$ & $29 \cdot 4^{c}$ & $22 \cdot 1$ & $6 \cdot 8^{a}$ & $55 \cdot 6^{b}$ & $27 \cdot 0^{c}$ \\
\hline 5th quintile & 14.6 & $26 \cdot 3^{\mathrm{a}}$ & $6 \cdot 0^{\mathrm{b}}$ & $5 \cdot 3^{\mathrm{b}}$ & $19 \cdot 1$ & $34 \cdot 1^{\mathrm{a}}$ & $10 \cdot 8^{\mathrm{b}}$ & $5 \cdot 7^{\mathrm{C}}$ \\
\hline \multicolumn{9}{|l|}{ Sweets } \\
\hline 1st quintile & $19 \cdot 3$ & $3.9^{\mathrm{a}}$ & $20 \cdot 0^{\mathrm{b}}$ & $34 \cdot 6^{c}$ & $20 \cdot 7$ & $6 \cdot 9^{a}$ & $21 \cdot 2^{b}$ & $35 \cdot 5^{c}$ \\
\hline 5th quintile & 19.7 & $33 \cdot 1^{a}$ & $24 \cdot 7^{\mathrm{a}}$ & $5 \cdot 0^{\mathrm{b}}$ & $17 \cdot 8$ & $28 \cdot 8^{a}$ & $20 \cdot 4^{\mathrm{b}}$ & $5 \cdot 0^{\mathrm{C}}$ \\
\hline \multicolumn{9}{|l|}{ Soft drinks } \\
\hline 1st quintile & $21 \cdot 0$ & $4 \cdot 0^{\mathrm{a}}$ & $26 \cdot 5^{b}$ & $36 \cdot 5^{c}$ & $18 \cdot 4$ & $2 \cdot 8^{a}$ & $27 \cdot 2^{b}$ & $32 \cdot 3^{b}$ \\
\hline 5th quintile & 18.0 & $33 \cdot 5^{a}$ & $17 \cdot 2^{b}$ & $2 \cdot 8^{\mathrm{C}}$ & $20 \cdot 4$ & $37 \cdot 2^{\mathrm{a}}$ & $20 \cdot 4^{b}$ & $2 \cdot 4^{\mathrm{C}}$ \\
\hline \multicolumn{9}{|l|}{ Salty pastry } \\
\hline 1st quintile & $18 \cdot 2$ & $8 \cdot 6^{\mathrm{a}}$ & $49 \cdot 8^{b}$ & $18 \cdot 7^{\mathrm{c}}$ & $20 \cdot 2$ & $10 \cdot 9^{a}$ & $47 \cdot 6^{b}$ & $20 \cdot 8^{c}$ \\
\hline 5th quintile & 3.8 & $6 \cdot 5^{\mathrm{a}}$ & $5 \cdot 1^{\mathrm{a}}$ & $0.8^{\mathrm{b}}$ & 4.0 & $6 \cdot 7^{\mathrm{a}}$ & $5 \cdot 6^{\mathrm{a}}$ & $0.6^{\mathrm{b}}$ \\
\hline
\end{tabular}

LC, latent class; EDF, Energy-Dense Foods dietary pattern.

${ }^{*}$ Proportions were compared by $x^{2}$ test considering Bonferroni's correction for post hoc multiple comparisons. Unlike superscript letters indicate significant differences between dietary patterns at a significance level of $5 \%$.

†In order to avoid redundancy, reference category (2nd to 4th quintiles, combined) is not presented.

Prospective relationships between dietary patterns and adiposity are presented according to the conceptual model depicted in Fig. 1. The association of dietary patterns at 4 years of age with adiposity 3 years later (association (a) in Fig. 1; Table 3) was modified by child's sex. The interaction term was statistically significant in the BMI $Z$-score model $(P=0.046)$ and was consistent in other models (FMI, $P=0.053$; WHtR, $P=0.063$ ). Hence, we chose to show final models stratified by child's sex. In crude models (model 1, Table 3), girls following the EDF dietary pattern at 4 years of age, when compared with those following the Healthier pattern, had significantly higher BMI $Z$-scores ( $\beta=0 \cdot 142 ; 95 \%$ CI 0.029, 0.256), FMI $(\beta=0 \cdot 108 ; 95 \%$ CI $0 \cdot 008,0 \cdot 209)$ and $\operatorname{WHtR}(\beta=0 \cdot 136 ; 95 \%$ CI $0 \cdot 036,0 \cdot 237)$, while in boys these associations were not significant. After adjustment for maternal (education, BMI) 
Table 2 Dietary patterns at 7 years of age: proportions of children in lower and higher consumption categories by sex $(n 3473)^{\star}$, Generation XXI prospective cohort study (Porto, Portugal, 2005-2006)

\begin{tabular}{|c|c|c|c|c|c|c|c|c|}
\hline \multirow[b]{2}{*}{ Item/category† } & \multicolumn{4}{|c|}{ Girls } & \multicolumn{4}{|c|}{ Boys } \\
\hline & $\begin{array}{l}\text { Total sample } \\
\text { (n 1722) }\end{array}$ & $\begin{array}{c}\text { LC1 } \\
\text { (EDF) } \\
(n 840)\end{array}$ & $\begin{array}{c}\text { LC2 } \\
\text { (Snacking) } \\
(n \text { 174) }\end{array}$ & $\begin{array}{c}\text { LC3 } \\
\text { (Healthier) } \\
(n 708)\end{array}$ & $\begin{array}{c}\text { Total sample } \\
\text { (n 1751) }\end{array}$ & $\begin{array}{c}\text { LC1 } \\
\text { (EDF) } \\
(n \text { 922) }\end{array}$ & $\begin{array}{c}\text { LC2 } \\
\text { (Snacking) } \\
(n 170)\end{array}$ & $\begin{array}{c}\text { LC3 } \\
\text { (Healthier) } \\
(n \text { 659) }\end{array}$ \\
\hline \multicolumn{9}{|l|}{ Fruit } \\
\hline 1st quintile & $40 \cdot 5$ & $50 \cdot 1^{a}$ & $74 \cdot 1^{\mathrm{b}}$ & $20 \cdot 8^{c}$ & 39.7 & $49 \cdot 2^{a}$ & $67 \cdot 6^{b}$ & $19 \cdot 1^{c}$ \\
\hline 5th quintile & $2 \cdot 8$ & $2 \cdot 3^{a}$ & $1 \cdot 7^{a}$ & $3 \cdot 7^{a}$ & 4.5 & $3 \cdot 8^{a}$ & $1 \cdot 8^{a}$ & $6 \cdot 1^{a}$ \\
\hline \multicolumn{9}{|l|}{ Vegetable soup } \\
\hline$\leq 1 / d$ & 41.6 & $55 \cdot 7^{\mathrm{a}}$ & $47 \cdot 7^{\mathrm{a}}$ & $23 \cdot 3^{b}$ & $42 \cdot 7$ & $54.0^{a}$ & $45 \cdot 9^{a}$ & $25 \cdot 9^{b}$ \\
\hline$\geq 2 / d$ & 58.4 & $44 \cdot 3^{a}$ & $52 \cdot 3^{a}$ & $76 \cdot 7^{\mathrm{a}}$ & $57 \cdot 3$ & $46 \cdot 0^{a}$ & $54 \cdot 1^{a}$ & $74 \cdot 1^{\mathrm{b}}$ \\
\hline \multicolumn{9}{|c|}{ Vegetables on a plate } \\
\hline 1st quintile & 23.4 & $22 \cdot 9^{a}$ & $69 \cdot 5^{\mathrm{b}}$ & $12 \cdot 7^{\mathrm{c}}$ & $26 \cdot 9$ & $27 \cdot 2^{a}$ & $75.9^{b}$ & $13 \cdot 8^{\mathrm{c}}$ \\
\hline 5th quintile & $24 \cdot 2$ & $21 \cdot 4^{a}$ & $4 \cdot 6^{\mathrm{b}}$ & $32 \cdot 2^{c}$ & $22 \cdot 1$ & $17 \cdot 4^{a}$ & $2 \cdot 9^{b}$ & $33 \cdot 7^{c}$ \\
\hline \multicolumn{9}{|l|}{ Milk } \\
\hline 1st quintile & 63.4 & $63 \cdot 3^{\mathrm{a}}$ & $68 \cdot 4^{\mathrm{a}}$ & $62 \cdot 3^{\mathrm{a}}$ & $59 \cdot 0$ & $60 \cdot 7^{a}$ & $57 \cdot 6^{\mathrm{a}}$ & $56 \cdot 9^{\mathrm{a}}$ \\
\hline 5th quintile & $3 \cdot 0$ & $3 \cdot 1^{\mathrm{a}}$ & $4 \cdot 6^{\mathrm{a}}$ & $2 \cdot 5^{\mathrm{a}}$ & $2 \cdot 2$ & $2 \cdot 4^{\mathrm{a}}$ & $0.6^{\mathrm{a}}$ & $2 \cdot 4^{\mathrm{a}}$ \\
\hline \multicolumn{9}{|l|}{ Yoghurt } \\
\hline 1st quintile & 69.5 & $63 \cdot 1^{\mathrm{a}}$ & $69 \cdot 0^{a, b}$ & $77 \cdot 3^{b}$ & $64 \cdot 8$ & $62 \cdot 0^{a}$ & $54.1^{\mathrm{a}}$ & $71 \cdot 3^{\mathrm{a}}$ \\
\hline 5th quintile & 1.3 & $1 \cdot 7^{\mathrm{a}}$ & $1 \cdot 1^{\mathrm{a}}$ & $0.8^{a}$ & 1.9 & $2 \cdot 2^{\mathrm{a}}$ & $2 \cdot 9^{a}$ & $1 \cdot 2^{a}$ \\
\hline \multicolumn{9}{|l|}{ Cheese } \\
\hline 1st quintile & 29.8 & $24 \cdot 4^{\mathrm{a}}$ & $71.8^{\mathrm{b}}$ & $26 \cdot 0^{\mathrm{a}}$ & $30 \cdot 4$ & $25 \cdot 8^{a}$ & $67 \cdot 1^{\mathrm{b}}$ & $27 \cdot 5^{a}$ \\
\hline 5th quintile & 6.9 & $8 \cdot 8^{a}$ & $1 \cdot 7^{\mathrm{b}}$ & $3 \cdot 8^{\mathrm{b}}$ & $6 \cdot 2$ & $8 \cdot 0^{a}$ & $5 \cdot 3^{a, b}$ & $3.9^{b}$ \\
\hline \multicolumn{9}{|l|}{ Fish } \\
\hline 1st quintile & $51 \cdot 7$ & $63 \cdot 3^{\mathrm{a}}$ & $73 \cdot 6^{\mathrm{b}}$ & $32 \cdot 6^{\mathrm{C}}$ & $51 \cdot 1$ & $62 \cdot 7^{\mathrm{a}}$ & $71.8^{\mathrm{a}}$ & $29 \cdot 6^{\mathrm{b}}$ \\
\hline 5th quintile & $26 \cdot 4$ & $18 \cdot 0^{a}$ & $19 \cdot 5^{a}$ & $38 \cdot 1^{b}$ & $29 \cdot 3$ & $21 \cdot 4^{a}$ & $20 \cdot 0^{a}$ & $42 \cdot 8^{b}$ \\
\hline \multicolumn{9}{|l|}{ Meat and eggs } \\
\hline 1st quintile & 34.5 & $34 \cdot 4^{\mathrm{a}}$ & $47 \cdot 1^{b}$ & $31 \cdot 5^{a}$ & $31 \cdot 1$ & $29 \cdot 2^{a}$ & $45 \cdot 9^{b}$ & $30 \cdot 0^{a}$ \\
\hline 5th quintile & 6.7 & $8 \cdot 8^{\mathrm{a}}$ & $7 \cdot 5^{\mathrm{a}, \mathrm{b}}$ & $4 \cdot 1^{\mathrm{b}}$ & 6.5 & $9 \cdot 4^{a}$ & $6 \cdot 5^{a}$ & $2 \cdot 4^{\mathrm{b}}$ \\
\hline \multicolumn{9}{|l|}{ Processed meat } \\
\hline 1st quintile & 22.9 & $7 \cdot 3^{a}$ & $65 \cdot 5^{b}$ & $30.9^{c}$ & $22 \cdot 6$ & $8 \cdot 8^{a}$ & $67 \cdot 6^{b}$ & $30 \cdot 3^{c}$ \\
\hline 5th quintile & $28 \cdot 7$ & $38 \cdot 1^{a}$ & $13 \cdot 2^{b}$ & $21 \cdot 3^{c}$ & $27 \cdot 9$ & $34 \cdot 4^{\mathrm{a}}$ & $14 \cdot 1^{b}$ & $22 \cdot 3^{b}$ \\
\hline \multicolumn{9}{|c|}{ Rice/potatoes/pasta } \\
\hline$\leq 1 / d$ & $18 \cdot 5$ & $19 \cdot 4^{a}$ & $37 \cdot 4^{b}$ & $12 \cdot 9^{c}$ & $16 \cdot 5$ & $15 \cdot 7^{a}$ & $38 \cdot 2^{b}$ & $12 \cdot 0^{a}$ \\
\hline$\geq 2 / d$ & 81.5 & $80 \cdot 6^{a}$ & $62 \cdot 6^{\mathrm{b}}$ & $87 \cdot 1^{\mathrm{C}}$ & 83.5 & $84 \cdot 3^{a}$ & $61 \cdot 8^{b}$ & $88 \cdot 0^{a}$ \\
\hline \multicolumn{9}{|l|}{ Bread } \\
\hline 1st quintile & $39 \cdot 3$ & $37.0^{\mathrm{a}}$ & $54 \cdot 6^{b}$ & $38 \cdot 1^{a}$ & $30 \cdot 8$ & $28 \cdot 2^{a}$ & $40 \cdot 0^{b}$ & $32 \cdot 2^{a, b}$ \\
\hline 5th quintile & 2.5 & $3 \cdot 3^{a}$ & $1 \cdot 1^{\mathrm{a}}$ & $1 \cdot 8^{\mathrm{a}}$ & 3.4 & $4 \cdot 4^{\mathrm{a}}$ & $4 \cdot 7^{\mathrm{a}, \mathrm{b}}$ & $1.7^{\mathrm{b}}$ \\
\hline \multicolumn{9}{|l|}{ Crisps } \\
\hline 1st quintile & $26 \cdot 0$ & $8 \cdot 0^{\mathrm{a}}$ & $24 \cdot 7^{b}$ & $47 \cdot 6^{c}$ & $22 \cdot 8$ & $8 \cdot 4^{\mathrm{a}}$ & $24 \cdot 1^{b}$ & $42 \cdot 6^{c}$ \\
\hline 5th quintile & $8 \cdot 1$ & $13 \cdot 9^{a}$ & $10 \cdot 3^{a}$ & $0.7^{\mathrm{b}}$ & $7 \cdot 8$ & $12 \cdot 8^{a}$ & $10 \cdot 6^{a}$ & $0.0^{\mathrm{b}}$ \\
\hline \multicolumn{9}{|l|}{ Pizza/burger } \\
\hline 1st quintile & $7 \cdot 3$ & $1.5^{\mathrm{a}}$ & $30 \cdot 5^{b}$ & $8 \cdot 5^{c}$ & $5 \cdot 8$ & $2 \cdot 0^{\mathrm{a}}$ & $28 \cdot 8^{b}$ & $5 \cdot 3^{c}$ \\
\hline \multirow{2}{*}{\multicolumn{9}{|c|}{ Sweets }} \\
\hline & & & & & & & & \\
\hline 1st quintile & $35 \cdot 7$ & $16 \cdot 4^{a}$ & $35 \cdot 1^{\mathrm{b}}$ & $58 \cdot 8^{c}$ & $36 \cdot 1$ & $19 \cdot 0^{a}$ & $35 \cdot 3^{b}$ & $60 \cdot 5^{c}$ \\
\hline 5th quintile & $7 \cdot 3$ & $12 \cdot 0^{\mathrm{a}}$ & $9 \cdot 2^{\mathrm{a}}$ & $1 \cdot 1^{\mathrm{b}}$ & 8.3 & $12 \cdot 5^{\mathrm{a}}$ & $12 \cdot 9^{a}$ & $1 \cdot 2^{\mathrm{b}}$ \\
\hline \multicolumn{9}{|l|}{ Soft drinks } \\
\hline 1st quintile & $13 \cdot 7$ & $2 \cdot 0^{\mathrm{a}}$ & $17 \cdot 2^{\mathrm{b}}$ & $26 \cdot 7^{\mathrm{C}}$ & 11.7 & $1.5^{\mathrm{a}}$ & $17 \cdot 1^{\mathrm{b}}$ & $24 \cdot 4^{\mathrm{b}}$ \\
\hline 5th quintile & $23 \cdot 6$ & $39 \cdot 8^{a}$ & $26 \cdot 4^{b}$ & $3.7^{c}$ & $27 \cdot 7$ & $42 \cdot 5^{a}$ & $28 \cdot 2^{b}$ & $6 \cdot 8^{\mathrm{c}}$ \\
\hline \multicolumn{9}{|l|}{ Salty pastry } \\
\hline 1st quintile & $4 \cdot 8$ & $2 \cdot 5^{a}$ & $13 \cdot 8^{b}$ & $5 \cdot 4^{c}$ & $4 \cdot 3$ & $2 \cdot 1^{\mathrm{a}}$ & $17 \cdot 1^{b}$ & $4 \cdot 2^{c}$ \\
\hline 5th quintile & $36 \cdot 7$ & $55 \cdot 5^{a}$ & $38.5^{b}$ & $14.0^{c}$ & 36.5 & $54 \cdot 2^{a}$ & $34 \cdot 1^{b}$ & $12 \cdot 3^{c}$ \\
\hline
\end{tabular}

LC, latent class; EDF, Energy-Dense Foods dietary pattern.

${ }^{*}$ Proportions were compared by $X^{2}$ test considering Bonferroni's correction for post hoc multiple comparisons. Unlike superscript letters indicate significant differences between dietary patterns at a significance level of $5 \%$.

†In order to avoid redundancy, reference category (2nd to 4 th quintiles, combined) is not presented.

and child's characteristics (any breast-feeding, physical exercise, screen time, exact age and adiposity at 4 years of age; model 2, Table 3), these associations were attenuated (BMI Z-score: $\beta=0.075 ; 95 \%$ CI $0.009, \quad 0.140 ; \quad$ FMI: $\beta=0.071 ; 95 \%$ CI $0.000,0 \cdot 142$; WHtR: $\beta=0.094 ; 95 \%$ CI $0 \cdot 023,0 \cdot 164)$, but retained statistical significance.

Associations of adiposity measures at 4 years of age with dietary patterns at 7 years of age are shown in Table 4 (association (b) in Fig. 1). In both crude and adjusted models, none of the adiposity measures at 4 years of age was associated with subsequent dietary patterns in girls or boys.

Dietary patterns tracked across the two ages (association (c) in Fig. 1; results in Table 5). The EDF pattern at 4 years significantly increased the odds of children following the same pattern at 7 years in both girls $(\mathrm{OR}=4.48 ; 95 \% \mathrm{CI}$ $3 \cdot 51,5 \cdot 70)$ and boys (OR=4.70; $95 \%$ CI 3.68, 5.98). 
Table 3 Association of dietary patterns at 4 years of age with adiposity at 7 years of age by sex $(n 3473)^{\star}$, Generation XXI prospective cohort study (Porto, Portugal, 2005-2006)

\begin{tabular}{|c|c|c|c|c|c|c|c|}
\hline \multirow[b]{2}{*}{ Adiposity at 7 years $\dagger$} & \multirow{2}{*}{$\begin{array}{l}\text { Dietary pattern } \\
\text { at } 4 \text { years }\end{array}$} & \multicolumn{3}{|c|}{ Girls (n 1722) } & \multicolumn{3}{|c|}{ Boys ( $n$ 1751) } \\
\hline & & $n$ & $\beta$ & $95 \% \mathrm{Cl}$ & $n$ & $\beta$ & $95 \% \mathrm{Cl}$ \\
\hline \multicolumn{8}{|l|}{ BMI Z-score } \\
\hline \multirow[t]{3}{*}{ Model $1 \ddagger$} & Healthier & 754 & Ref. & & 721 & Ref. & \\
\hline & EDF & 753 & $0 \cdot 142$ & $0.029,0.256$ & 780 & -0.014 & $-0.135,0.107$ \\
\hline & Snacking & 215 & -0.147 & $-0.317,0.023$ & 250 & -0.125 & $-0.297,0.047$ \\
\hline \multirow[t]{3}{*}{ Model $2 \S$} & Healthier & 754 & Ref. & & 721 & Ref. & \\
\hline & EDF & 753 & 0.075 & $0.009,0.140$ & 780 & -0.014 & $-0.093,0.065$ \\
\hline & Snacking & 215 & -0.010 & $-0.105,0.085$ & 250 & -0.087 & $-0.197,0.023$ \\
\hline \multicolumn{8}{|l|}{ FM\% } \\
\hline \multirow[t]{3}{*}{ Model 1‡ } & Healthier & 754 & Ref. & & 721 & Ref. & \\
\hline & EDF & 753 & 0.082 & $-0.019,0.183$ & 780 & -0.033 & $-0.134,0.068$ \\
\hline & Snacking & 215 & -0.068 & $-0.220,0.083$ & 250 & -0.016 & $-0.160,0.128$ \\
\hline \multirow[t]{3}{*}{ Model $2 \S$} & Healthier & 754 & Ref. & & 721 & Ref. & \\
\hline & EDF & 753 & 0.053 & $-0.171,0.058$ & 780 & 0.008 & $-0.077,0.092$ \\
\hline & Snacking & 215 & -0.057 & $-0.171,0.058$ & 250 & -0.035 & $-0.153,0.083$ \\
\hline \multicolumn{8}{|c|}{ 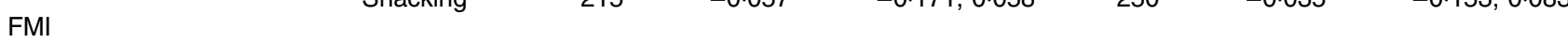 } \\
\hline \multirow[t]{3}{*}{ Model 1‡ } & Healthier & 754 & Ref. & & 721 & Ref. & \\
\hline & EDF & 753 & $0 \cdot 108$ & $0.008,0.209$ & 780 & -0.024 & $-0.125,0.077$ \\
\hline & Snacking & 215 & -0.058 & $-0.210,0.093$ & 250 & -0.026 & $-0.170,0.11 \varepsilon$ \\
\hline \multirow[t]{3}{*}{ Model $2 \S$} & Healthier & 754 & Ref. & & 721 & Ref. & \\
\hline & EDF & 753 & 0.071 & $0.000,0.142$ & 780 & 0.012 & $-0.067,0.090$ \\
\hline & Snacking & 215 & -0.044 & $-0.147,0.059$ & 250 & -0.043 & $-0.153,0.066$ \\
\hline \multicolumn{8}{|l|}{ WHtR } \\
\hline \multirow[t]{3}{*}{ Model $1 \ddagger$} & Healthier & 754 & Ref. & & & Ref. & \\
\hline & EDF & 753 & 0.136 & $0.036,0.237$ & 721 & 0.015 & $-0.087,0.116$ \\
\hline & Snacking & 215 & -0.067 & $-0.218,0.084$ & 780 & 0.011 & $-0.133,0.155$ \\
\hline \multirow[t]{3}{*}{ Model $2 \S$} & Healthier & 754 & Ref. & & 250 & Ref. & \\
\hline & EDF & 753 & 0.094 & $0.023,0.164$ & 721 & 0.049 & $-0.028,0.125$ \\
\hline & Snacking & 215 & -0.007 & $-0.109,0.096$ & 780 & -0.019 & $-0.125,0.087$ \\
\hline
\end{tabular}

$\beta$, regression coefficient; EDF, Energy-Dense Foods dietary pattern; FM\%, fat mass percentage; FMI, fat mass index; WHtR, waist-to-height ratio; Ref., reference category.

${ }^{*}$ Results correspond to association (a) in Fig. 1. Statistically significant associations are highlighted in bold.

†All adiposity measures are in Z-score units. BMI Z-scores were defined according to the WHO's age- and sex-specific standards. FM\%, FMI and WHtR were converted into $Z$-scores using the sex-specific means and standard deviations observed in the study sample.

¥Crude.

§Adjusted for maternal (education, BMI) and child (any breast-feeding, physical exercise, screen time, exact age and each adiposity measure at 4 years) characteristics.

Table 4 Association of adiposity measures at 4 years of age with dietary patterns at 7 years of age by sex $(n 3473)^{\star}$, Generation XXI prospective cohort study (Porto, Portugal, 2005-2006)

\begin{tabular}{|c|c|c|c|c|c|c|c|c|}
\hline \multirow[b]{3}{*}{ Adiposity at 4 years $\dagger$} & \multicolumn{4}{|c|}{ Dietary patterns at 7 years in girls ( $n$ 1722) $\ddagger$} & \multicolumn{4}{|c|}{ Dietary patterns at 7 years in boys ( $n$ 1751) $\ddagger$} \\
\hline & \multicolumn{2}{|c|}{$\operatorname{EDF}(n 840)$} & \multicolumn{2}{|c|}{ Snacking ( $n$ 174) } & \multicolumn{2}{|c|}{ EDF ( $n$ 922) } & \multicolumn{2}{|c|}{ Snacking $(n$ 170) } \\
\hline & OR & $95 \% \mathrm{Cl}$ & OR & $95 \% \mathrm{Cl}$ & OR & $95 \% \mathrm{Cl}$ & OR & $95 \% \mathrm{Cl}$ \\
\hline \multicolumn{9}{|l|}{ BMI Z-score } \\
\hline Model $1 \S$ & 1.02 & $0.93,1.12$ & 0.95 & $0.82,1.11$ & 1.08 & $0.98,1.19$ & 1.01 & $0.86,1.19$ \\
\hline Model 2ll & 0.96 & $0.86,1.07$ & 0.94 & $0.80,1.11$ & 1.03 & $0.93,1.15$ & 0.99 & $0.83,1.18$ \\
\hline \multicolumn{9}{|l|}{ FM\% } \\
\hline Model $1 \S$ & 0.97 & $0.87,1.07$ & 0.99 & $0.84,1.17$ & 1.02 & $0.92,1.12$ & 1.03 & $0.87,1.22$ \\
\hline Model 2\| & 0.94 & $0.84,1.05$ & 0.94 & $0.78,1.12$ & 1.05 & $0.94,1.18$ & 1.02 & $0.84,1.22$ \\
\hline \multicolumn{9}{|l|}{ FMI } \\
\hline Model $1 \S$ & 0.98 & $0.88,1.08$ & 0.97 & $0.82,1.15$ & 1.05 & $0.95,1.16$ & 1.04 & $0.88,1.23$ \\
\hline Model 2ll & 0.93 & $0.83,1.04$ & 0.91 & $0.76,1.09$ & 1.06 & $0.94,1.19$ & 1.02 & $0.84,1.22$ \\
\hline \multicolumn{9}{|l|}{ WHtR } \\
\hline Model $1 \S$ & 0.96 & $0.87,1.06$ & 0.94 & $0.80,1 \cdot 12$ & 1.00 & $0.91,1.11$ & 1.04 & $0.88,1.23$ \\
\hline Model 2\|l & 0.93 & $0.83,1.04$ & 0.91 & $0.76,1.09$ & 1.01 & $0.90,1.14$ & 1.03 & $0.85,1.23$ \\
\hline
\end{tabular}

EDF, Energy-Dense Foods dietary pattern; FM\%, fat mass percentage; FMI, fat mass index; WHtR, waist-to-height ratio.

${ }^{\star}$ Results correspond to association (b) in Fig. 1.

†All adiposity measures are in Z-score units. BMI Z-scores were defined according to the WHO's age- and sex-specific standards. FM\%, FMI and WHtR were converted into $Z$-scores using the sex-specific means and standard deviations observed in the study sample.

$\ddagger$ Reference category (healthier dietary pattern at 7 years) not shown in order to avoid redundancy.

$\S$ Crude.

IIdjusted for maternal (education, BMI) and child (any breast-feeding, physical exercise practice, daily screen time, exact age and dietary pattern at 4 years) characteristics. 
Table 5 Association of dietary patterns at 4 years of age with dietary patterns at 7 years of age by sex $(n 3473)^{\star}$, Generation XXI prospective cohort study (Porto, Portugal, 2005-2006)

\begin{tabular}{|c|c|c|c|c|c|c|c|c|c|c|c|c|c|}
\hline \multirow[b]{3}{*}{ Dietary pattern at 4 years } & \multicolumn{6}{|c|}{ Dietary pattern at 7 years in girls $(n$ 1722) $\dagger$} & \multirow{3}{*}{$\begin{array}{l}\text { Dietary pattern } \\
\text { at } 4 \text { years }\end{array}$} & \multicolumn{6}{|c|}{ Dietary pattern at 7 years in boys $(n 1751) \dagger$} \\
\hline & \multicolumn{3}{|c|}{ EDF $(n 840)$} & \multicolumn{3}{|c|}{ Snacking ( $n$ 174) } & & \multicolumn{3}{|c|}{ EDF $(n$ 922) } & \multicolumn{3}{|c|}{ Snacking $(n 170)$} \\
\hline & $n$ & OR & $95 \% \mathrm{Cl}$ & $n$ & OR & $95 \% \mathrm{Cl}$ & & $n$ & OR & $95 \% \mathrm{Cl}$ & $n$ & OR & $95 \% \mathrm{Cl}$ \\
\hline \multicolumn{14}{|l|}{ Model 1‡ } \\
\hline Healthier ( $n$ 754) & 234 & Ref. & & 57 & Ref. & & Healthier ( $n$ 721) & 247 & Ref. & & 48 & Ref. & \\
\hline EDF $(n 753)$ & 515 & $4 \cdot 48$ & $3 \cdot 51,5 \cdot 70$ & 55 & $1 \cdot 84$ & $1 \cdot 20,2 \cdot 83$ & EDF $(n 780)$ & 563 & $4 \cdot 68$ & $3 \cdot 68,5.97$ & 47 & 2.04 & $1 \cdot 30,3 \cdot 21$ \\
\hline Snacking ( $n$ 215) & 91 & $2 \cdot 38$ & $1 \cdot 64,3 \cdot 43$ & 62 & $6 \cdot 00$ & $3 \cdot 88,9 \cdot 54$ & Snacking ( $n$ 250) & 112 & $2 \cdot 34$ & $1 \cdot 64,3.36$ & 63 & $8 \cdot 39$ & $5 \cdot 28,13 \cdot 32$ \\
\hline \multicolumn{14}{|l|}{ Model $2 \S$} \\
\hline Healthier $(n 754)$ & 234 & Ref. & & 57 & Ref. & & Healthier ( $n$ 721) & 247 & Ref. & & 48 & Ref. & \\
\hline $\operatorname{EDF}(n 753)$ & 515 & $4 \cdot 48$ & $3 \cdot 52,5 \cdot 71$ & 55 & 1.85 & $1 \cdot 21,2 \cdot 84$ & $\operatorname{EDF}(n 780)$ & 563 & $4 \cdot 70$ & $3 \cdot 68,5.98$ & 47 & 2.04 & $1 \cdot 30,3 \cdot 20$ \\
\hline Snacking (n 215) & 91 & $2 \cdot 36$ & $1 \cdot 63,3 \cdot 41$ & 62 & 5.94 & $3 \cdot 73,9 \cdot 46$ & Snacking (n 250) & 112 & $2 \cdot 35$ & $1 \cdot 64,3 \cdot 37$ & 63 & $8 \cdot 39$ & $5 \cdot 27,13 \cdot 31$ \\
\hline
\end{tabular}

EDF, Energy-Dense Foods dietary pattern; Ref., reference category.

${ }^{*}$ Results correspond to association (c) in Fig. 1. Statistically significant associations are highlighted in bold.

†Reference category (healthier dietary pattern at 7 years) not shown in order to avoid redundancy.

$\ddagger$ Model 1 is adjusted for maternal (education, BMI) and child (any breast-feeding, physical exercise practice, daily screen time and exact age) characteristics. $\S$ Model 2 includes variables in Model 1 and is further adjusted for BMI Z-score at child's age of 4 years.

Likewise, the Snacking pattern persisted in time in both sexes.

Adiposity measures also tracked across the two ages (association (d) in Fig. 1; results in Table 6). In girls, each increase of $1 Z$-score unit in BMI at 4 years significantly increased later BMI by 0.829 (95\% CI 0.802, 0.857) Z-score units; in boys, an increase of $1 Z$-score unit in BMI at 4 years was associated with a significant increase of 0.851 (95\% CI 0.817, 0.885) Z-score units in BMI at 7 years of age.

\section{Discussion}

Present findings indicate that dietary patterns as well as adiposity measures persist from 4 to 7 years in both sexes, and that an EDF dietary pattern high in sweets, sugarsweetened beverages, salty pastry and processed meat followed at pre-school age is significantly positively associated with measures of total and central adiposity in 7-year-old girls, but not in boys.

Dietary patterns identified in the current study are comparable to those identified in other reports. Among pre-school children from the $\mathrm{UK}^{(36,37)}$ three dietary patterns were identified at 3 and 4 years of age: a 'traditional' pattern characterized by meat and vegetables; a 'junk' pattern characterized by high-fat processed foods (comparable to the present EDF pattern); and a 'health conscious' pattern rich in vegetarian-style foods, cheese and fish (comparable to the current Healthier pattern). Additionally, snacking dietary patterns - partly comparable to the current Snacking pattern - were also identified at 3 years of age in children from the $\mathrm{UK}^{(36)}$ and in 5-12-year-old children from Colombia $^{(38)}$. Two other studies identified dietary patterns comparable to the current EDF pattern; a 'Processed and fast foods' pattern was identified at multiple time points among French children ${ }^{(39)}$ and a 'Snacky' pattern was identified in Greek 4-year-olds ${ }^{(40)}$.
Table 6 Association of adiposity measures across the two ages by sex $(n 3473)^{*}$, Generation XXI prospective cohort study (Porto, Portugal, 2005-2006)

\begin{tabular}{|c|c|c|c|c|}
\hline \multirow{3}{*}{$\begin{array}{l}\text { Adiposity at } \\
4 \text { years } \dagger\end{array}$} & \multicolumn{4}{|c|}{ Adiposity at 7 years } \\
\hline & \multicolumn{2}{|c|}{ Girls (n 1722) } & \multicolumn{2}{|c|}{ Boys ( $n$ 1751) } \\
\hline & $\beta$ & $95 \% \mathrm{Cl}$ & $\beta$ & $95 \% \mathrm{Cl}$ \\
\hline BMI Z-score & & & & \\
\hline $\begin{array}{l}\text { Model } 1 \ddagger \\
\text { Model } 2 \S \\
\text { FM\% }\end{array}$ & $\begin{array}{l}0.831 \\
0.829\end{array}$ & $\begin{array}{l}0.803,0.858 \\
0.802,0.857\end{array}$ & $\begin{array}{l}0.852 \\
0.851\end{array}$ & $\begin{array}{l}0.817,0.886 \\
0.817,0.885\end{array}$ \\
\hline $\begin{array}{l}\text { Model } 1 \ddagger \\
\text { Model } 2 \S\end{array}$ & $\begin{array}{l}0.640 \\
0.640\end{array}$ & $\begin{array}{l}0.604,0.676 \\
0.604,0.676\end{array}$ & $\begin{array}{l}0.573 \\
0.573\end{array}$ & $\begin{array}{l}0.534,0.611 \\
0.535,0.611\end{array}$ \\
\hline FMI & & & & \\
\hline $\begin{array}{l}\text { Model 1‡ } \\
\text { Model } 2 \S \\
\text { WHtR }\end{array}$ & $\begin{array}{l}0.709 \\
0.709\end{array}$ & $\begin{array}{l}0.677,0.741 \\
0.676,0.741\end{array}$ & $\begin{array}{l}0.639 \\
0.639\end{array}$ & $\begin{array}{l}0.603,0.674 \\
0.604,0.675\end{array}$ \\
\hline $\begin{array}{l}\text { Model } 1 \ddagger \\
\text { Model } 2 \S\end{array}$ & $\begin{array}{l}0.711 \\
0.711\end{array}$ & $\begin{array}{l}0.679,0.744 \\
0.678,0.743\end{array}$ & $\begin{array}{l}0.661 \\
0.663\end{array}$ & $\begin{array}{l}0.627,0.696 \\
0.628,0.698\end{array}$ \\
\hline
\end{tabular}

$\beta$, regression coefficient; FM\%, fat mass percentage; FMI, fat mass index; WHtR, waist-to-height ratio.

${ }^{*}$ Results correspond to association (d) in Fig. 1. Statistically significant associations are highlighted in bold.

†All adiposity measures are in Z-score units. BMI Z-scores were defined according to the WHO's age- and sex-specific standards. FM\%, FMI and $\mathrm{WHtR}$ were converted into $Z$-scores using the sex-specific means and standard deviations observed in the study sample.

¥Model 1 is adjusted for maternal (education, BMI) and child (any breastfeeding, physical exercise practice, daily screen time and exact age) characteristics.

$\S$ Model 2 is further adjusted for dietary pattern at child's age of 4 years.

Tracking of dietary patterns is also comparable to reports from other studies ${ }^{(13,39)}$. In the current sample, the odds of staying in the EDF pattern across the two ages were considerably higher than the odds of changing into the Snacking pattern and vice versa. Still, some interchangeability between these two patterns was observed and some children changed from the Snacking into the EDF pattern (girls, 42\%; boys 45\%). Keeping in mind that the 'snack' pattern identified among 3-year-olds from the $\mathrm{UK}^{(22)}$ was 
not identified at 4,7 or 9 years of age, it is possible that these 'snack'/'snacking' patterns are still reflecting a transition to the family diet and that - with increasing age children tend to change into other patterns.

Considering the association of dietary patterns with adiposity, the 'junk' dietary pattern identified in 3-yearolds from the $\mathrm{UK}^{(12)}$ was not associated with obesity at 7 years of age and the 'energy-dense, low-fibre, high-fat' pattern practised at 5 years was not significantly associated with fat mass at 7 years, although this pattern practised at 7 years was significantly positively associated with adiposity at 9 years of age ${ }^{(13)}$. As these studies did not report sex-specific associations, comparison with the present findings is hampered. Regarding other ages, in a previous study conducted in our cohort from 2 to 4 years of age ${ }^{(41)}$, we did not find evidence of effect modification by sex on the association between consumption of EDF and BMI. In contrast, among adolescents from the USA, slight sex differences have been reported: a 'starchy foods' pattern was negatively associated with overweight in boys, while no association was found in girls ${ }^{(42)}$.

The positive association between the EDF dietary pattern and adiposity found in girls may be explained by the sexual dimorphism in adiposity that emerges before the onset of puberty $^{(17,43)}$, with girls showing higher relative body fat and abdominal subcutaneous adipose tissue compared with boys ${ }^{(44)}$. An earlier adiposity rebound reported in girls $^{(18)}$ could also explain the present findings.

Considering the possibility of distinct BMI trajectories and distinct susceptibilities to certain environmental factors in girls and boys, prospective associations could hypothetically - differ according to sex. Hence, the conceptual model presented in Fig. 1 was used in order to examine if such differences were observed. Yet there was no evidence of distinct prospective associations.

It should be highlighted that adjustment for BMI at 4 years of age diminished the magnitude of the association between the EDF dietary pattern and adiposity observed in girls. This suggests that, in girls, the EDF dietary pattern has a direct effect on later adiposity, but also an indirect effect mediated through adiposity at the age of 4 years. In a sensitivity analysis, we fitted models excluding children who were overweight at age 4 years and results were similar (data not shown), giving strength to the current findings. If the observed effect modification by sex is true, the present results may be reflecting the higher propensity to store body fat and higher risk of obesity reported in females ${ }^{(15,16,20)}$. However, given that males and females are both susceptible to obesity, and that girls may have an earlier adiposity rebound, it is possible that the EDF pattern is positively associated with adiposity in boys at later stages.

Evaluation of distinct measures of adiposity is a strength of the present study. Since BMI is not able to distinguish between lean and fat mass, and the accuracy of FM\% depends on height and cannot be assessed independently of free-fat mass, it is important to use an index adjusted for height (i.e. FMI) ${ }^{(45)}$. Since these measures do not indicate regional fat distribution, it was also crucial to assess a measure of central fatness (i.e. WHtR) as abdominal adiposity is associated with increased metabolic complications in children ${ }^{(46)}$.

The use of latent class analysis to identify dietary patterns is an advantage, as this methodology is especially well suited for categorical variables asymmetrically distributed, common in FFQ. Latent class analysis focuses on relationships between individuals and describes how the probabilities of a set of observed categorical variables (e.g. FFQ items) vary across individuals, finding the smallest number of unobserved variables (i.e. latent classes) that distinguish homogeneous clusters of individuals (e.g. patterns). It has the advantage of avoiding subjective cut-off points on underlying dimensions, as it adds classes stepwise until the model fits the data well and the classification is provided directly by the model $^{(14)}$

Restricting the analysis of dietary patterns at 7 years to the consumption categories observed at 4 years deserves discussion. This approach was defined a priori to assess if children switched between dietary patterns from 4 to 7 years of age, rather than to identify new patterns at 7 years. Considering that median probabilities of class membership supported an acceptable classification of individuals into each latent class at both ages (data not shown), this approach was deemed appropriate.

Besides the prospective design and children's age frame, another strength of the current study was its sufficient statistical power to assess the interaction of child's sex on the association between diet and adiposity. In addition, as children were part of a large population-based cohort regularly followed, several potential confounding factors could be assessed.

Assessment of dietary intake by FFQ could be argued as a limitation. However, comparison with $3 \mathrm{~d}$ food diaries supported a reasonable validity and reliability of the FFQ data. Also, the EDF pattern was significantly higher in energy than the Snacking and Healthier patterns (girls, $6765 v .6304$ and $6576 \mathrm{~kJ}$; boys, $7175 v .6735$ and $6910 \mathrm{~kJ})$.

Inclusion of a sub-sample could have introduced some bias into the analysis. A previous study conducted in this cohort $^{(47)}$ managed missing data using full-information maximum likelihood estimation and comparison of this model with the model excluding missing values suggested that substantial bias was not expected (Cohen's $\kappa=0 \cdot 85$ ). Hence, it is not expected that alternative methods to manage missing data would result in different conclusions. In addition, as Cohen's effect size values are not high $(<0 \cdot 35)$, the slight differences between this sample and the remaining cohort are likely due to large sample size rather than to substantial differences between participants $^{(48)}$. 


\section{Conclusion}

Children practising unhealthier dietary patterns at the age of 4 years were more likely to follow the unhealthier dietary patterns 3 years later. Likewise, higher adiposity persisted across the two ages in both girls and boys. In contrast, a dietary pattern high in EDF was consistently positively associated with adiposity 3 years later only in girls. Future studies should further examine if sex modifies the association between diet and adiposity in distinct populations at different ages, with particular focus on underlying metabolic pathways. Present findings suggest that interventions to prevent unhealthy dietary habits should focus on early dietary patterns and that girls in particular may need early attention.

\section{Acknowledgements}

Acknowledgements: The authors gratefully acknowledge the families enrolled in Generation XXI for their kindness, all members of the research team for their enthusiasm and perseverance, and the participating hospitals and their staff for their help and support. Financial support: Generation XXI was funded by Programa Operacional de Saúde (Regional Department of Ministry of Health). It has support from the Portuguese Foundation for Science and Technology (FCT) and from the Calouste Gulbenkian Foundation. This study was supported through FEDERCOMPETE and through national funding from the FCT (Portuguese Ministry of Education and Science) within the project PTDC/SAU-EPI/121532/2010 (grant number FCOMP-01-0124-FEDER-021177). The work of C.D. was also supported by the FCT (grant number SFRH/BD/81788/ 2011). The funders had no role in the design, analysis or writing of this article. Conflict of interest: None. Authorship: C.D. performed statistical analyses, drafted the initial manuscript and contributed to the interpretation of data. M.S. carried out statistical analysis and contributed to the interpretation of data. A.O. contributed to the design of data collection instruments and contributed to the interpretation of data. P.M. and A.G. contributed to the interpretation of data. H.B. conceptualized and designed the study, coordinated and supervised data collection and contributed to the interpretation of data. C.L. contributed to the design of study, contributed to the design of data collection instruments and contributed to the interpretation of data. All authors contributed to the concept of the study, critically reviewed the manuscript and approved the final submitted version. Ethics of human subject participation: The study complied with the ethical principles expressed in the Declaration of Helsinki and was approved by the University of Porto Medical School/São João Hospital Centre ethics committee. Signed informed consent according to the Helsinki Declaration was required for all participants.

\section{References}

1. Olds T, Maher C, Zumin S, Péneau S et al. (2011) Evidence that the prevalence of childhood overweight is plateauing: data from nine countries. Int J Pediatr Obes 6, 342-360.

2. Wabitsch M, Moss A \& Kromeyer-Hauschild K (2014) Unexpected plateauing of childhood obesity rates in developed countries. BMC Med 12, 17.

3. Lobstein T, Jackson-Leach R, Moodie M et al. (2015) Child and adolescent obesity: part of a bigger picture. Lancet $\mathbf{3 8 5}$, 2510-2520.

4. Ahrens W, Pigeot I, Pohlabeln H et al. (2014) Prevalence of overweight and obesity in European children below the age of 10. Int J Obes (Lond) 38, Suppl. 2, S99-S107.

5. Wijnhoven T, van Raaij J, Spinelli A et al. (2014) WHO European Childhood Obesity Surveillance Initiative: body mass index and level of overweight among 6-9-year-old children from school year 2007/2008 to school year 2009/ 2010. BMC Public Health 14, 806.

6. Cole T \& Lobstein T (2012) Extended international (IOTF) body mass index cut-offs for thinness, overweight and obesity. Pediatr Obes 7, 284-294.

7. de Onis M, Onyango A, Borghi E et al. (2007) Development of a WHO growth reference for school-aged children and adolescents. Bull World Health Organ 85, 660-667.

8. Hu FB (2002) Dietary pattern analysis: a new direction in nutritional epidemiology. Curr Opin Lipidol 13, 3-9.

9. Román-Viñas B, Barba LR, Ngo J et al. (2009) Validity of dietary patterns to assess nutrient intake adequacy. Br J Nutr 101, Suppl. 2, S12-S20.

10. Ambrosini GL (2014) Childhood dietary patterns and later obesity: a review of the evidence. Proc Nutr Soc 73, 137-146.

11. Durão C, Oliveira A, Santos A et al. (2017) Protein intake and dietary glycemic load of 4-year-olds and association with adiposity and serum insulin at 7 years of age: sexnutrient and nutrient-nutrient interactions. Int J Obes (Lond) 41, 533-541.

12. Reilly J, Armstrong J, Dorosty A et al. (2005) Early life risk factors for obesity in childhood: cohort study. BMJ 330, 1357.

13. Johnson L, Mander A, Jones L et al. (2008) Energy-dense, low-fiber, high-fat dietary pattern is associated with increased fatness in childhood. Am J Clin Nutr 87, 846-854.

14. Muthén B \& Muthén L (2000) Integrating person-centered and variable-centered analyses: growth mixture modeling with latent trajectory classes. Alcohol Clin Exp Res 24, 882-891.

15. Wells J (2007) Sexual dimorphism of body composition. Best Pract Res Clin Endocrinol Metab 21, 415-430.

16. Power M \& Schulkin J (2008) Sex differences in fat storage, fat metabolism, and the related health risks from obesity: possible evolutionary origins. Br J Nutr 99, 931-940.

17. Karlsson A-K, Kullberg J, Stokland E et al. (2013) Measurements of total and regional body composition in preschool children: a comparison of MRI, DXA, and anthropometric data. Obesity (Silver Spring) 21, 1018-1024.

18. Wen X, Kleinman K, Gillman M et al. (2012) Childhood body mass index trajectories: modeling, characterizing, pairwise correlations and socio-demographic predictors of trajectory characteristics. BMC Med Res Methodol 12, 38.

19. Silverwood R, De Stavola B, Cole T et al. (2009) BMI peak in infancy as a predictor for later BMI in the Uppsala Family Study. Int J Obes (Lond) 33, 929-937.

20. Wisniewski A \& Chernausek S (2009) Gender in childhood obesity: family environment, hormones, and genes. Gend Med 6, Suppl. 1, 76-85.

21. Mikkila V, Rasanen L, Raitakari OT et al. (2005) Consistent dietary patterns identified from childhood to adulthood: the Cardiovascular Risk in Young Finns Study. Br J Nutr 93, 923-931. 
22. Northstone K \& Emmett P (2008) Are dietary patterns stable throughout early and mid-childhood? A birth cohort study. Br J Nutr 100, 1069-1076.

23. Waters E, Silva-Sanigorski A, Burford BJ et al. (2011) Interventions for preventing obesity in children. Cochrane Database Syst Rev issue 12, CD001871.

24. Larsen PS, Kamper-Jørgensen M, Adamson A et al. (2013) Pregnancy and birth cohort resources in Europe: a large opportunity for aetiological child health research. Paediatr Perinat Epidemiol 27, 393-414.

25. Moreira I, Severo M, Oliveira A et al. (2014) Social and health behavioural determinants of maternal child-feeding patterns in preschool-aged children. Matern Child Nutr 12, 314-325.

26. Durão C, Andreozzi V, Oliveira A et al. (2015) Maternal child-feeding practices and dietary inadequacy of 4-year-old children. Appetite 92, 15-23.

27. Lopes C, Aro A, Azevedo A et al. (2007) Intake and adipose tissue composition of fatty acids and risk of myocardial infarction in a male Portuguese community sample. $J \mathrm{Am}$ Diet Assoc 107, 276-286.

28. Durão C, Severo M, Oliveira A et al. (2017) Association of maternal characteristics and behaviors with 4-year-old children's dietary patterns. Matern Child Nutr 13, e12278.

29. World Health Organization (2006) WHO Child Growth Standards: Length/Height-for-Age, Weight-for-Age, Weightfor-Length, Weight-for-Height and Body Mass Indexfor-Age: Methods and Development, pp 223-227. Geneva: WHO.

30. Kyle U, Bosaeus I, De Lorenzo A et al. (2004) Bioelectrical impedance analysis - part II: utilization in clinical practice. Clin Nutr 23, 1430-1453.

31. Schaefer F, Georgi M, Zieger A et al. (1994) Usefulness of bioelectric impedance and skinfold measurements in predicting fat-free mass derived from total body potassium in children. Pediatr Res 35, 617-624.

32. Harrington D, Staiano A, Broyles S et al. (2013) Waist circumference measurement site does not affect relationships with visceral adiposity and cardiometabolic risk factors in children. Pediatr Obes 8, 199-206.

33. Severo M, Gaio AR, Lourenço P et al. (2012) Diagnostic value of patterns of symptoms and signs of heart failure: application of latent class analysis with concomitant variables in a cross-sectional study. BMJ Open 2, e001510.

34. Liu M \& Hancock G (2014) Unrestricted mixture models for class identification in growth mixture modeling. Educ Psychol Meas 74, 557-584.

35. Vermunt JK \& Magidson J (2002) Latent class cluster analysis. In Applied Latent Class Models, pp 89-106 [J Hagenaars and A McCutcheon, editors]. Cambridge: Cambridge University Press.
36. North K \& Emmett P (2000) Multivariate analysis of diet among three-year-old children and associations with socio-demographic characteristics. Eur J Clin Nutr 54, 73-80.

37. Northstone K \& Emmett P (2005) Multivariate analysis of diet in children at four and seven years of age and associations with socio-demographic characteristics. Eur J Clin Nutr 59, 751-760.

38. Shroff M, Perng W, Baylin A et al. (2014) Adherence to a snacking dietary pattern and soda intake are related to the development of adiposity: a prospective study in school-age children. Public Health Nutr 17, 1507-1513.

39. Lioret S, Betoko A, Forhan A et al. (2015) Dietary patterns track from infancy to preschool age: cross-sectional and longitudinal perspectives. J Nutr 145, 775-782.

40. Leventakou V, Sarri K, Georgiou V et al. (2015) Early life determinants of dietary patterns in preschool children: Rhea mother-child cohort, Crete, Greece. Eur J Clin Nutr 70, 60-65.

41. Durão C, Severo M, Oliveira A et al. (2015) Evaluating the effect of energy-dense foods consumption on preschool children's body mass index: a prospective analysis from 2 to 4 years of age. EurJ Nutr 54, 835-843.

42. Cutler G, Flood A, Hannan P et al. (2012) Association between major patterns of dietary intake and weight status in adolescents. Br J Nutr 108, 349-356.

43. Staiano A, Broyles S, Gupta A et al. (2013) Ethnic and sex differences in visceral, subcutaneous, and total body fat in children and adolescents. Obesity (Silver Spring) 21, 1251-1255.

44. Barreira T, Broyles S, Gupta A et al. (2014) Relationship of anthropometric indices to abdominal and total fat in youth: sex and race differences. Obesity (Silver Spring) 22, $1345-1350$.

45. Liu P, Ma F, Lou $\mathrm{H}$ et al. (2013) The utility of fat mass index vs. body mass index and percentage of body fat in the screening of metabolic syndrome. BMC Public Health 13, 629 .

46. McCarthy H \& Ashwell M (2006) A study of central fatness using waist-to-height ratios in UK children and adolescents over two decades supports the simple message - 'keep your waist circumference to less than half your height. Int J Obes (Lond) 30, 988-992.

47. Teixeira C, Silva S, Severo M et al. (2015) Socioeconomic position early in adolescence and mode of delivery later in life: findings from a Portuguese birth cohort. PLoS One 10, $\mathrm{e} 0119517$.

48. Husted JA, Cook RJ, Farewell VT et al. (2000) Methods for assessing responsiveness: a critical review and recommendations. J Clin Epidemiol 53, 459-468. 